\title{
La construcción social de gramáticas juveniles: reflexiones sobre la desigualdad a través de estudios longitudinales ${ }^{1}$
}

Milena Arancibiaª

Ana Miranda ${ }^{3}$

Resumen: El artículo se propone aportar al debate teórico sobre la construcción de la desigualdad social a través de un estudio sobre trayectorias sociales de hombres y mujeres en base al análisis de resultados de investigación del Programa Gramáticas de la Juventud (FLACSO Argentina). Aborda entrevistas retrospectivas realizadas con personas jóvenes que alcanzaron la mayoría de edad durante una de las peores crisis sociales de la Argentina. De forma sustantiva, presenta el debate sobre la intersección espacio y géneros, en tanto claves para la reflexión sobre la construcción social de gramáticas juveniles. Sus conclusiones brindan elementos de importancia para la elaboración de políticas de juventud y empleo.

Palabras claves: juventud, desigualdad espacial, género, estudios longitudinales.

1 Esta investigación recibió financiamiento del Proyecto Construcción de autonomía y desigualdad social: tendencias hacia el cambio y la reproducción en las transiciones juveniles de principios del siglo 21. PIP 2014 CONICET

2 Facultad Latinoamericana de Ciencias Sociales (FLACSO), Ciudad de Buenos Aires, Argentina - Consejo Nacional de Investigaciones Científicas y Técnicas (CONICET) - Ciudad de Buenos Aires - Argentina marancibia@flacso.org.ar

3 Facultad Latinoamericana de Ciencias Sociales (FLACSO), Ciudad de Buenos Aires, Argentina - Consejo Nacional de Investigaciones Científicas y Técnicas (CONICET) - Ciudad de Buenos Aires - Argentina amiranda@flacso.org.ar 


\section{The social construction of youth grammars: reflections on inequality through longitudinal studies}

Abstract: The article aims to contribute to the theoretical debate on the construction of social inequality through a research on men and women's trajectories, based on the results of the Grammar of Youth Research Program (FLACSO Argentina). We analyze retrospective interviews with young people who reached the age of majority during the worst social crisis in Argentina. Substantially, it presents the debate on the intersection of space and genres, as keys for reflection on the social construction of youth grammars. Its conclusions provide important elements for the development of youth and employment policies.

Keywords: youth, spatial inequality, gender, longitudinal studies.

\section{Introducción}

Existe una fuerte tradición de investigación centrada en el estudio de las culturas juveniles con una corriente de gran acervo que pondera las especificidades locales en la construcción identitaria (Cerbino, 2012; Chaves, 2005; Reguillo, 2000; Valenzuela Arce, 2015; entre otros). Se han desarrollado estudios sobre la intersección de las identidades juveniles y el espacio, que abordaron los procesos de fragmentación territorial y su impacto en la vida cotidiana (Bayon, 2015; Saraví, 2015; entre otros). No obstante, pesar de cierta acumulación de investigaciones en la región que analizan las trayectorias juveniles desde una perspectiva de género, es escaso el desarrollo de aquellas que abordan la dimensión espacial de las trayectorias de modo comparativo entre mujeres y varones y entre sectores sociales.

El desarrollo de estudios que abordan la identidad, su relación con el tiempo, el espacio y el género entre las y los jóvenes aporta claves para comprender el proceso de reproducción de la desigualdad en los países de la región. Aunque las trayectorias están fuertemente influenciadas por las oportunidades disponibles en los espacios y grupos familiares donde transcurren sus biografías, los estudios de juventud continúan centrados en la transición a la vida adulta a través del acceso al empleo. Desde un punto de vista teórico, la centralidad del empleo en los procesos de transición evade el debate sobre la magnitud de las tareas de cuidado y sobre la estigmatización de quienes desarrollan transiciones donde el empleo es informal, inestable o inexistente.

Basados en las experiencias de las y los jóvenes, una serie de estudios ha profundizado en las nociones de identidad, espacio y género. Entre ellos, algunos 
abordaron el concepto de arraigo, que facilita la incorporación de la identidad generacional, las raíces y la afectividad como nociones centrales para el análisis de la transición juvenil. Este concepto es particularmente útil cuando se abordan las trayectorias de las mujeres y varones jóvenes en Argentina, y especialmente las trayectorias de aquellas y aquellos que viven en lugares donde la centralidad del empleo productivo es inexistente (Miranda; Arancibia, 2017). Al considerar el enfoque del arraigo, recurrimos a Cuervo y Wyn donde las “(...) interacciones con familiares, amigos, vecinos y otros miembros de la comunidad tienen la capacidad de generar un sentido de pertenencia" (Cuervo; Wyn, 2014: 7) y tienen efectos en los procesos de cambio y reproducción de la estructura de oportunidades y acceso al bienestar.

En la misma dirección, la propuesta conceptual de gramáticas de la juventud propone estudiar tanto los espacios que contextualizan y determinan las experiencias de las y los jóvenes en diferentes campos, como sus formas de acción (agencia) en relación a estas estructuras y determinaciones. De manera análoga a la conceptualización de habitus, sostiene que las gramáticas juveniles constituyen principios de acción socialmente estructurados que permiten múltiples biografías, de acuerdo con patrones y valores jerarquizados (Bourdieu, 2008). La noción de gramática intenta hacer visible la existencia de un sistema de reglas que organiza el curso de la vida a través de la cual las y los jóvenes interactúan y negocian durante su transición a la vida adulta. Este sistema de reglas tiene un anclaje territorial y evoluciona de una manera social y culturalmente situada (Bendit; Miranda, 2017).

Estudiar los espacios vividos que contextualizan y contribuyen a delinear las experiencias de los jóvenes en distintos aspectos de la vida, brinda también elementos claves en el debate sobre la construcción de femineidades y masculinidades. La categoría de género no sólo aborda los papeles sociales resultantes de la división sexual del trabajo sino que "incluye dimensiones sociales, psíquicas y sexuales que se entrelazan de forma compleja” (Segato, 2010: 77). Esta forma de abordaje busca comprender la mecánica que organiza las relaciones de poder entre las identidades femeninas y masculinas, es decir el soporte ideológico del patriarcado que sustenta la jerarquía de prestigio de comportamientos y roles.

En este artículo se abordan las experiencias y representaciones de las y los jóvenes sobre los lugares que habitan, en los que se encuadran sus recorridos y que aportan a comprender las desiguales posibilidades de participación económica, social y cultural de mujeres y varones de distintos sectores sociales. En particular, la noción de arraigo al lugar constituye el eje para analizar la influencia del espacio en las trayectorias sociales de los jóvenes. La misma remite al 
vínculo que establecen los jóvenes con su lugar de residencia, basado en aspectos afectivos y en otros más racionalizados.

A partir de una investigación longitudinal realizada en zonas de la ciudad donde habitan sectores populares y otras donde habitan sectores predominantemente de clase media del Área Metropolitana de Buenos Aires, este artículo se propone abordar la dimensión espacial de las trayectorias desde una perspectiva de género, puntualizando las diferencias entre las experiencias de mujeres y varones.

El texto presenta resultados del Programa de Estudios Longitudinales Gramáticas de la Juventud que se desarrolla en la Facultad Latinoamericana de Ciencias Sociales (FLACSO Argentina), que aborda cómo las mujeres y los varones que nacieron a principios de los 80 experimentaron sus trayectorias juveniles en un contexto de crisis y recuperación económica. Las historias se corresponden con un trabajo de campo realizado entre 2013 y 2017, cuando las y los jóvenes ingresaban a la cuarta década de su vida. Las siguientes preguntas guiaron la investigación: ¿Cómo se manifiesta la influencia del espacio en la construcción identitaria de mujeres y varones jóvenes de diferentes clases sociales? ¿Cuál es la relación entre el espacio social y el arraigo en las trayectorias juveniles? ¿Cómo influyen las diferentes escalas espaciales y el arraigo en las trayectorias de trabajo y en las familiares? ¿Cómo se manifiesta de forma diferente el arraigo en mujeres y varones?

Con la interpelación de estas preguntas, el artículo está organizado en cuatro secciones. La primera sección presenta las especificidades del contexto latinoamericano, específicamente el crecimiento económico y la continuidad de la fragmentación territorial. La segunda sección presenta la estrategia metodológica de la investigación. En la tercera sección, siguiendo a Hopkins (2015), se aborda la importancia de las escalas espaciales en la definición de las trayectorias juveniles, y también muestra el desarrollo de la relación entre género y arraigo en las trayectorias de jóvenes de diferentes sectores sociales. Finalmente, en las conclusiones, se compara el arraigo de las mujeres y los varones que viven en la misma ciudad pero en condiciones muy desiguales con el propósito de contribuir a comprender cómo las posiciones se interrelacionan y, en algunos casos las desigualdades existentes se refuerzan mutuamente, profundizando la segmentación.

\section{Transiciones juveniles en América Latina}

En América Latina, la desigualdad se manifiesta en el acceso a bienes básicos como la educación y la salud, en la brecha de ingresos, y en un fuerte proceso de fragmentación urbana que influye en el desarrollo de la construcción de 
proyectos de vida de las y los jóvenes. En la región existen amplias problemáticas sociales, vinculadas a la extensión de la desigualdad social y también con la violencia y la exclusión juvenil (Valenzuela Arce, 2015) que ameritan marcos conceptuales propios. En efecto, a pesar de las tendencias redistributivas de las primeras décadas de 2000, la estructuración de un modelo de ciudad neoliberal tendió a profundizar las desigualdades, estimulando procesos segregatorios (Rodríguez et al., 2007).

Algunos estudios han sostenido que se ha extendido un proceso de fragmentación basado en las estrategias de cierre social de los sectores de mayores ingresos. La difusión de esta división implica la expansión de fronteras, la conformación de espacios mutuamente excluyentes y la ruptura de vínculos entre los diferentes segmentos sociales (Saraví, 2015). La financiarización de la renta urbana generó un proceso de redistribución regresiva de la tierra y las oportunidades urbanas asociadas a ella, lo que resultó en la promoción de experiencias generacionales altamente diferenciadas entre los diferentes grupos sociales, y desde edades tempranas. La fragmentación espacial tiene fuertes consecuencias en la vida cotidiana de los jóvenes.

Estos procesos, que se han extendido a todos los países de la región, se presentan con diferente intensidad en las ciudades y países latinoamericanos. En Argentina, un país con ingresos intermedios, después de dos décadas de desempleo acentuado, el cambio en la estrategia económica implicó que el trabajo volviera a adquirir centralidad en las relaciones sociales. Las políticas macroeconómicas heterodoxas y las políticas de inclusión social generaron un extenso programa de protección social, pero con poco impacto en el posicionamiento laboral de las mujeres, quienes, si bien fueron cubiertas por los programas de ingresos públicos asociados con la maternidad, continuaron a cargo de las tareas de cuidado ${ }^{4}$.

La persistencia de una fuerte división sexual del trabajo cuestiona la transición juvenil centrada en el empleo y la autonomía de la vivienda, que parece ineficaz para el análisis de las transiciones femeninas, ya que representa un modelo donde no se tiene en cuenta el trabajo de cuidado y la reproducción ampliada de la vida. Muchas mujeres jóvenes que han crecido en hogares de bajos ingresos experimentan una prolongación entre la participación en las tareas de cuidado en su familia de origen y las actividades de cuidado en su propio hogar, a menudo sin tener acceso al empleo independientemente de las

4 En 2013, las mujeres tienen cifras muy por encima de los hombres en horas dedicadas al trabajo de cuidado (88,9\% para el primero frente al 58,2\%) (Rodríguez Enríquez; Marzonetto, 2015). 
estrategias reproductivas de la familia (Marco Novarro, 2013; Rodríguez Enríquez; Marzonetto, 2015).

La contracara del modelo tradicional de división sexual del trabajo antes mencionado impone a los varones la cultura de la provisión, y la responsabilidad de la inserción laboral también desde edades tempranas, la cual ha sido abordada desde distintas investigaciones sobre jóvenes y masculinidad (Fraiman; Rossal, 2009; Perelman; Vargas, 2013; Kessler, 2012; Ward, 2014). Pero los varones de distintos sectores sociales tienen desigual acceso al empleo o actividades económicas. Uno de los factores que influye en las posibilidades de acceso al mercado laboral es el lugar de residencia. En este sentido, se analizará la relación entre el espacio y las trayectorias juveniles específicamente entre mujeres y varones que habitan en barrios con diferentes características del Área Metropolitana de Buenos Aires.

En Argentina, el proceso de fragmentación espacial es menos intenso que en otras naciones del continente latinoamericano; sin embargo, se considera que la fragmentación espacial tiene fuertes implicancias en la vida cotidiana de las y los jóvenes. La subjetividad de mujeres y hombres se encuentra atravesada por la segmentación social y se expresa a través de decisiones relacionadas con la familia, la educación y el trabajo durante la transición a la vida adulta. Como parte de este proceso, las y los jóvenes experimentan una variedad de sentimientos de arraigo al lugar, algunos impulsados por la elección, otros afianzados en los grupos familiares o comunitarios. En la siguiente sección, presentamos la estrategia de investigación longitudinal utilizada para la construcción de los datos.

\section{El Programa Gramáticas de la Juventud}

Gramáticas de la Juventud es un programa de investigación que comenzó en el año 1998 a desarrollar estudios longitudinales mediante la aplicación de encuestas y entrevistas en profundidad. La metodología que emplea es el análisis de cohortes, aplicada a jóvenes de diferentes generaciones. La información compilada permite analizar las principales transformaciones en las transiciones juveniles en Argentina desde principios del siglo XXI. Actualmente, cuenta con información de dos generaciones que alcanzaron la mayoría de edad (18 años) en coyunturas económicas y sociales muy diferentes. La primera cohorte longitudinal (denominada G99) agrupa a jóvenes que nacieron entre 1981 y 1982 y que alcanzaron los 18 años en un período marcado por la aplicación de programas de ajuste, desempleo y recesión económica (1999). La segunda cohorte (G11) agrupa a jóvenes que nacieron entre 1993 y 1994 y que alcanzaron los 18 
años de edad en un contexto caracterizado por mayores oportunidades de empleo y protección social (2011).

La información presentada en este artículo fue elaborada con la cohorte G99 a través de entrevistas retrospectivas en profundidad llevadas a cabo cuando las personas tenían entre 32 y 34 años. La selección de entrevistados corresponde a una muestra segmentada de acuerdo con el nivel educativo alcanzado ("hasta primario completo", "hasta secundario completo", "hasta superior completo"), el género (varón y mujer) y las características del hábitat, que incluye barrios relegados (tanto modalidades informales como diferentes tipos de viviendas sociales) y barrios predominantemente de clase media. La muestra se compone de cincuenta y dos jóvenes urbanos (mitad varones y mitad mujeres) de diferentes grupos sociales en el Área Metropolitana de Buenos Aires.

Las historias biográficas relevadas a lo largo del trabajo de campo permiten reconstruir el recorrido completo de la transición de los jóvenes entre la educación y el mundo del trabajo (ya sea como empleo o como trabajos de cuidado) sobre principios de la vida adulta de una generación que se había integrado en el mercado laboral en años de crisis económica, pero que luego vivieron un período de recuperación y expansión de derechos (Bendit; Miranda, 2016). El análisis presentado en este artículo se centra en la transición a la vida adulta de mujeres y varones de barrios relegados y de barrios predominantemente de la clase media, y reflexiona sobre la dimensión espacial de las trayectorias, el arraigo y su importancia en la construcción de rutas biográficas.

\section{Género, escalas espaciales y arraigo}

Durante los últimos años, colegas del ámbito de la sociología de la juventud han trabajado en abordajes conceptuales que permiten la incorporación de las nociones de identidad, género, espacio y arraigo (Cuervo; Wyn, 2014; Savage, 2010; Warr, 2015, entre otros). El análisis de las identidades y el arraigo entre las mujeres y los varones ha permitido comprender las decisiones tomadas en el proceso de construcción de sus proyectos de vida y las posibilidades con las que se encuentran en los recorridos. Como forma de contribuir a la acumulación de conocimiento sobre el Sur Global (Connell, 2007), dejando de lado el foco en el carácter normativo y norte-céntrico de las transiciones juveniles, se analizaron las trayectorias educativas y laborales de las mujeres y varones jóvenes en Argentina (Miranda; Arancibia, 2017). A través de la incorporación de las nociones de tiempo y espacio, que permitieron reflexionar sobre la identidad en el marco de cursos de vida heterogéneos y no lineales (Worth, 2015), se avanzó en 
un marco analítico más amplio para el análisis de la desigualdad en el contexto de la globalización en el mundo contemporáneo (Farrugia, 2014).

Las contribuciones de la geografía humana proporcionan herramientas para analizar las dimensiones simbólicas y espaciales de la fragmentación urbana (Bayón; Saravi, 2017) y su impacto en las transiciones de las mujeres y los varones. Cada vez más, la dimensión espacial de la vida social configura el acceso a redes y recursos diferenciados entre los jóvenes de diferentes sectores sociales, donde se construyen identidades femeninas y masculinas altamente diferenciadas. En tiempos de globalización, estos vínculos se presentan en escalas espaciales diferenciadas según las áreas de conectividad, que están enmarcadas por el acceso de los hogares a los recursos económicos y sociales.

Hopkins enfatiza la importancia de considerar las escalas espaciales no solo como un marco, sino también como el resultado de un proceso, argumentando que "los lugares utilizados, habitados y ocupados por los jóvenes son importantes y a menudo tienen una fuerte influencia en la configuración de sus identidades personales" (Hopkins, 2015: 762, traducción propia). Como una forma de estudiar los espacios que contextualizan y determinan las experiencias de los jóvenes en diferentes ámbitos, y contribuir al debate sobre las transiciones juveniles, el concepto de gramáticas de la juventud expresa la preocupación sobre la relación entre las estructuras de actividades que las sociedades contemporáneas ofrecen a los jóvenes, y los marcos normativos que respaldan las expectativas puestas en el cumplimiento. Estas expectativas se basan en un sistema de valores jerárquicos, sobre los cuales se construye un sistema de premios y castigos que estigmatiza a aquellos y aquellas que desarrollan sus transiciones por fuera de las actividades y recorridos hegemónicos.

Uno de los desafíos que presenta este artículo es vincular la conceptualización de gramáticas juveniles, arraigo y escalas espaciales para analizar las trayectorias de mujeres y varones jóvenes que viven en el Área Metropolitana de Buenos Aires, la principal aglomeración urbana de Argentina. El estudio de las escalas espaciales es de gran importancia, ya que dan forma a experiencias ampliamente diversas de transición y de vínculo con el espacio. En este sentido, se presenta la idea de una comparación entre distintas formas de arraigo al lugar. La primera forma llamada "arraigo electivo" es flexible, líquida y fluida. Siguiendo a Savage, el arraigo electivo se entiende como "la forma en que las personas de clase media reclaman los derechos morales sobre el lugar a través de su capacidad de trasladarse a un lugar especifico o echar raíces en un lugar que no es funcionalmente importante para ellos, sino que importa simbólicamente" (Savage, 2010: 116, traducción propia). La segunda forma conocida como "fuerte arraigo territorial" (Mac Donald et al., 2005) es rígida y estable. 
A continuación, se presenta la evidencia empírica basada en los testimonios de mujeres y varones jóvenes que desarrollan sus recorridos biográficos en diferentes escalas espaciales. En primer lugar, se enfoca en las características de las historias de mujeres que viven en barrios relegados, su arraigo territorial y la influencia en la definición de sus trayectorias. Para mostrar el contraste, luego se concentra la mirada en las mujeres que habitan en barrios de clase media, enfatizando su arraigo al lugar en una la escala global. En segundo lugar, las historias biográficas de varones de barrios relegados remiten a un escenario marcado por el desigual acceso a los bienes urbanos y al mercado de trabajo. En oposición, las decisiones tomadas por aquellos varones con mayor capital económico, social y cultural expresan otras lógicas, entre las que se destaca el retraso de las transiciones por las expectativas asociadas al rol masculino.

\section{Trayectorias de mujeres que habitan en barrios relegados}

En países con escasa provisión estatal de servicios de bienestar, el cuidado se encuentra a cargo de las familias (Aguirre et al., 2014). En este escenario, las mujeres jóvenes están a cargo del sector de cuidado desde una edad temprana, lo que a menudo significa que sus trayectorias son de poca relación con el mercado laboral y los ingresos propios. El otro lado de la naturalización de las tareas de cuidado se expresa en la reificación del papel de la provisión entre los hombres, lo que evidencia el fuerte arraigo de la división sexual del trabajo (Fraser, 2016).

En el caso de las mujeres entrevistadas que habitan en barrios relegados, las decisiones vitales se llevan a cabo como parte de estrategias familiares, donde la colaboración mutua genera fuertes relaciones de interdependencia. Tal es el caso de Raquel, una joven de un barrio urbano informal (La Saladita, Avellaneda), que dejó la escuela secundaria en segundo año, para dedicarse a las tareas de cuidado de su familia. Su estrategia de supervivencia combinaba los ingresos provenientes de programas sociales con la venta de bienes ilegales y la ayuda de vecinos y familiares. Su testimonio registra las dificultades que tuvo a lo largo de su trayectoria para mantener un trabajo estable ya que ella tenía que cuidar sola a sus hijas porque su pareja pasaba la mayor parte del tiempo en la cárcel: "mis hijas conmigo para todos lados, por eso no trabajaba” (Raquel, comunicación personal, 2017). En cuanto a la relación con el barrio, en su relato se observan percepciones contradictorias. Por un lado, Raquel relataba los peligros de vivir en el barrio:

Ahí entrabas y no sabías si salías, y cuando salías no sabías si salías o si volvías bien a tu casa... (...) Cuando era chica no salía mucho yo, si bien era 
mucha la ausencia de mi mamá, mi papá trabajaba todo el día, mi hermana era muy sobreprotectora, ella no nos dejaba salir a ningún lado, porque era feo el barrio, ahí había muchos paraguayos donde si repente se agarraban y se peleaban, los paraguayos te cortaban todo, era ver mucha sangre, o que se agarraran a tiros. En ese sentido ella era muy chica, pero igual nos cuidaba como una leona, era más madre ella que mi viejo y mi vieja. Nos decía que nos quedemos ahí, que no salgamos, que esto, que lo otro, o sea trataba todo el tiempo de hacernos entender lo que afuera... no es que era malo, sino que había mucho peligro. Yo iba a la escuela y nos llevaba ella. Yo me acuerdo cuando íbamos al jardín, era a un par de cuadras largas, y yo tenía cuatro o cinco años y ella tenía nueve, diez años, y nos llevaba ella, era tan chiquita y nos llevaba a la mañana, y ella se volvía, los peligros que ella pasaba, porque ella nos cuidaba a nosotros (Raquel, comunicación personal, 2017).

Por otro lado, Raquel contaba que en una oportunidad le prestaron una vivienda en un barrio donde habitan predominantemente sectores de clase media del Conurbano Bonaerense (Villa Adelina), pero cuando realizó el movimiento residencial se sintió sola y ya no podía continuar con su participación en la cooperativa del barrio, por lo que dejó la vivienda que le habían prestado y regresó a la villa. En sus palabras:

Viste que son gente de guita los que viven en aquellos lados, era tipo zona residencial. La casa era hermosa... pero el trato de la gente, la gente de ahí es como que te ve y "ah, ¿vos quién sos?" (...) La casa era un encanto, que vos decís "no podes estar acá”... Y la terminé dejando... porque estaba sola. Ya ahí ya no venía a trabajar, no, no trabajaba.

Como se observa, en el momento en que intentó mudarse a un barrio de lo que se puede llamar la "ciudad formal", en el traslado ella perdió muchas de sus relaciones y recursos que la apoyaban en su vida diaria, tal como la ayuda de parientes, vecinos, pero también la posibilidad de participar del programa estatal de cooperativas en el que trabajaba, por lo que eligió regresar. En la elección de la vivienda no sólo se considera su funcionalidad como lugar de albergue y la accesibilidad a los bienes urbanos dados por la localización de ésta, sino que también adquiere centralidad el lugar de las relaciones sociales de afinidad (Cravino, 2009). Estas relaciones también constituyen los circuitos para acceder a los programas sociales, de los cuales, como subraya Raquel, se pierde la posibilidad de participar si se vive fuera del barrio. El arraigo territorial y su vínculo afectivo ejercen influencia, en gran medida, en 
la determinación de la trayectoria de Raquel, quien volvió a vivir a la villa en condiciones de privación extrema.

Los discursos de las entrevistadas están anclados en las raíces históricas y la historia familiar, reafirmando un arraigo y una identidad situada. En estos casos, la identidad se afirma en la disponibilidad vital para las tareas de cuidado y la naturaleza esencialista de la maternidad, que al tiempo que da cobijo, fortalece la reproducción de la división sexual del trabajo, en un marco de escasa movilidad social. Los afectos, las redes de sociabilidad barrial y el arraigo tienen fuerte peso en las decisiones. Como parte de una gramática juvenil local afirmada en la división de tareas enraizada en el género, las identidades femeninas disponibles se construyen a partir del arraigo a grupos familiares, comunitarios y territoriales. La perspectiva de la transición con la centralidad teórica del empleo productivo obstruye la reflexión sobre el proceso de reproducción social, donde las mujeres participan activamente.

\section{Trayectorias de mujeres que habitan en barrios de clase media}

Los datos recopilados sobre mujeres jóvenes que habían finalizado estudios secundarios y habitaban en barrios predominantemente de clase media, permiten analizar las transiciones que tienen lugar dentro de los parámetros socialmente estructurados para las y los jóvenes en un período de tiempo particular, y dentro de los modelos normativos hegemónicos. Una vez finalizada la escuela secundaria, durante la década de los 20 años, estas mujeres transitan la vida universitaria en combinación con diferentes experiencias de trabajo que, llegando a los 30, las posicionan en una situación de cierta estabilidad laboral (Miranda; Arancibia, 2017). En este grupo, se destacan las mujeres profesionales con trabajos "a tiempo completo", que logran autonomía y toman decisiones individualmente, es decir sin tantas ataduras con sus familias de origen. Podría decirse que durante este tiempo es donde la gramática de los jóvenes permite la afirmación de la carrera profesional y el consecuente aplazamiento de las tareas asociadas con la vida familiar. De hecho, el tiempo dedicado al estudio y al trabajo es inversamente proporcional a la formación de un grupo familiar propio, que se retrasa incluso después de los 30 años. Sin embargo, este proceso, que ocurre fuertemente entre mujeres que participan en una cultura de «juventud modernizada» (Bendit, 2008) no es libre de riesgos, ya que la configuración actual de los mercados laborales propone la postergación de la asunción de roles parentales hasta edades cada vez más avanzadas, involucrando muchas veces la medicalización de la actividad reproductiva (Fraser, 2016). 
Para abordar la dimensión espacial de las trayectorias de transición a la vida adulta de estas mujeres, específicamente la relación entre espacio e identidad, puede ser útil el concepto de arraigo electivo. Entre las entrevistadas está el caso de Bárbara, quien habitaba en un barrio de clase media de la Ciudad de Buenos Aires. Durante los primeros años de sus estudios universitarios tuvo trabajos temporales hasta que ingresó en un empleo estable, como parte de las experiencias que contribuyeron a su formación profesional. Poco antes de graduarse como psicóloga, consiguió un trabajo en el sector público, que al momento de la entrevista combinaba con la docencia universitaria y la práctica clínica en el ámbito privado. En sus primeros años de la cuarta década de su vida, Bárbara se encontraba realizando estudios de postgrado y entre sus expectativas futuras estaba tener hijos con su pareja. Además, planeaba mudarse a una vivienda con más espacio, pero sin cambiar de barrio, en el centro de la Ciudad de Buenos Aires, donde siempre había vivido. En sus propias palabras:

No me preguntes por qué pero nunca me imaginé lejos de la Universidad (...) hay una marcha y la miro desde la ventana, es 24 de marzo y me voy a la Plaza5. Me interesa la accesibilidad a cuestiones de la esfera política (Bárbara, comunicación personal, 2013).

En este sentido, es posible observar entre las mujeres entrevistadas que el arraigo al lugar está asociado a la importancia simbólica del lugar, que en este caso aparece ligada a la institución universitaria y a la posibilidad de participación en la vida política. En términos de Savage, la percepción sobre el lugar evidencia "el reconocimiento de que pueden elegir vivir en uno entre otros lugares, y que esta elección es muy reveladora y evocadora para ellos" (Savage, 2010: 118). Las trayectorias de estas mujeres, se construyen mediante aproximaciones sucesivas y tienen como característica central la privatización de la vida personal (Warr, 2015).

En el caso de la biografía de Susana, habitante de un barrio predominantemente de clase media de la Ciudad de Buenos Aires, había completado sus estudios superiores y trabajaba como fotógrafa de forma independiente. En su recorrido, la combinación de estudio y trabajo también contribuyó a la consolidación de su posición profesional. Su propensión a la movilidad, en

5 Los 24 de marzo, se realizan marchas en todo el país por el Día Nacional de la Memoria por la Verdad y la Justicia (denominación adoptada en el año 2001), en el que se conmemora el aniversario del golpe cívico-militar del año 1976, y se recuerda el Terrorismo de Estado, los crímenes de lesa humanidad cometidos por la dictadura y la desaparición forzada de personas. 
el marco de las ciudades globales, formaba parte de sus expectativas. En su relato, ella argumentaba:

Tengo una fantasía muy extraña: comprar un estudio e irme a Europa. Entonces, sabiendo que tengo un lugar al que regresar, puedo irme fácilmente. Podría irme a vivir a Barcelona, París... son las ciudades en las que más me imagino viviendo. O tal vez en Italia, pero no sé (Susana, comunicación personal, 2013).

Nuevamente, el vínculo con el lugar comunicado por Susana se muestra en imaginaciones que combinan el anclaje territorial y simbólico de forma electiva, que se refieren a sentimientos que tienen como punto de partida de gusto personal y la decisión consciente de moverse. El posicionamiento de la identidad se relaciona con un arraigo que se sostiene en los flujos globales, en ciudades altamente conectadas. La identidad de Susana se asocia con distinciones estéticas y de clase, como parte de una selección de elementos culturales (Cuervo; Wyn, 2017; Savage et al, 2005), que construyen una identidad femenina que difiere de aquella ligada a las tareas de cuidado familiar, al menos hasta los primeros años de la cuarta década de vida.

La reflexibilidad en la construcción de un arraigo electivo se construye en el marco de decisiones individualizadas y autocentradas, lo que permitirá la afirmación de un estilo de vida y consumo juvenil extendido (Savage, 2010). Luego de abordar algunas claves para comprender las desiguales trayectorias de las mujeres, se pasa la película de los recorridos biográficos masculinos.

\section{Varones, escalas espaciales y arraigo}

Si se analizan las trayectorias juveniles de los varones, se pueden observar características particulares. La contracara del modelo tradicional de división sexual del trabajo antes mencionado impone a los varones la cultura de la provisión, y la responsabilidad de la inserción laboral también desde edades tempranas, la cual ha sido abordada desde distintas investigaciones sobre jóvenes y masculinidad (Fraiman; Rossal, 2009), y en investigaciones sobre trabajo infantojuvenil (Marco Navarro, 2013). No obstante, los varones de diferentes sectores sociales tienen distinto acceso al empleo o actividades económicas, y entre los factores que producen mayores obstáculos y estigmatización se destaca el lugar de residencia (Mora Salas; Perez Sáinz, 2018). En base a estas hipótesis, en los apartados que siguen se analiza la relación entre el espacio y las trayectorias juveniles específicamente de varones que habitan en distintos lugares del Área Metropolitana de Buenos Aires. 


\section{Trayectorias de varones que habitan en barrios relegados}

Habitar la periferia puede implicar para los jóvenes varones distintas experiencias vinculadas a la desigualdad, algunas de ellas asociadas también a la violencia urbana y la estigmatización (Kessler, 2012). Estas experiencias, se expresan en contradicciones entre afirmación y estigmatización en la trayectoria de los jóvenes en asentamientos informales, como resultado de la espacialización de las relaciones de poder, cristalizada en las vidas cotidianas de las personas (Grimberg et al., 2019).

Daniel, habitante del asentamiento Mariló, San Miguel, había tenido una trayectoria educativo-laboral con una acumulación de experiencias diversas en empleos temporales de baja calificación y a sus 34 años se encontraba desempleado. Había habitado en distintas viviendas en barrios populares, pasó algunos años viviendo en situación de calle y también había realizado movimientos residenciales a otras provincias por un empleo que lo requería. Sin embargo, había regresado a la vivienda familiar por no tener otra opción. Cuando la inserción al mercado laboral es inestable y precaria, el barrio puede constituirse en un espacio de participación y una marca de la integración social. Él mismo identificaba su fuerte arraigo al lugar. Así se expresaba:

Es difícil a veces uno cuando crece cortar, es difícil desarraigarte de un lugar. Siempre vas a extrañar, yo anduve por muchos lados de la Argentina, trabajando, y nada... siempre volví a cruce Castelar, a San Miguel, por más que me haya ido, siempre volví. Vaya donde vaya me voy a querer volver. Soy sanmiguelino de corazón, hincha del Trueno Verde (Daniel, comunicación personal, 2017).

Como contracara del arraigo territorial se observa cómo opera el estigma en su trayectoria. En los recorridos de los jóvenes que viven en barrios relegados, el estigma y la criminalización son fuertes y se convierten en un factor de riesgo de exclusión y de exposición a la violencia policial (Saraví, 2004; Cravino, 2009; Kessler, 2012). En algunos casos se suma la discriminación étnica. Según su relato, había sido el estigma de "ser morocho" que lo había llevado a abandonar la escuela primaria, entrando luego en el consumo problemático de drogas.

El estigma se ve reproducido en la comparación con el habitante de "la Capital", es decir de la Ciudad de Buenos Aires. Así surge en el discurso un "otro" al que nunca podría parecerse. En la comparación que realiza el entrevistado, se hace evidente el estigma negativo que se adquiere al nacer en lugares relegados: 
E: ¿Qué representa el barrio para vos?

Principios. De no olvidarse de donde uno salió. Porque uno podría mudarse y no se olvida de donde uno salió. Pero a veces la gente sí, yo un par de veces me fui y me olvidé, y ahí fue cuando volví.

E: ¿Qué significa "saber de dónde uno salió"?

Y mucho...

E: ¿Qué?

En el sentido de que no te crezca el culo.

E: ¿En qué sentido?

En el sentido de que por ahí hoy en día estás viviendo en Capital y "ah no, ya sos nariz parada" y no sos así, siempre vas a ser la misma mierda...

E: ¿Como que uno puede cambiar si se va del barrio?

$\mathrm{Si}$, la misma gente te cambia. O las mismas amistades te quieren cambiar. Pero es cuestión de vos cambiar. A mí no me cambia nadie, mis hijos nada más me cambian.

E: ¿Pero pensás que cambiarías si te vas del barrio?

No!

Por detrás del relato de Daniel aparecen algunas de las imágenes construidas sobre los barrios relegados y sobre sus habitantes, presentes en la opinión pública y transmitidos por los medios de comunicación. Un imaginario hegemónico que construye una imagen de algunos barrios como subalternos, y que se construye sobre la comparación con otro espacio diferente, legítimo, estéticamente bello, habitable por aquellos que "lo merecen"6. En el discurso de Daniel, el arraigo al barrio aparece ligado a la comparación con un afuera, "la Capital", que refuerza la identidad barrial. La construcción de ese "otro", habitante de una ciudad distinta, con otras reglas, contribuye a construir un arraigo territorial fuerte.

La relación con ciertos espacios, como los espacios de trabajo, puede también aportar claves acerca de la construcción identitaria. Puede observarse el recorrido biográfico de Abel (barrio Ciudad Oculta, CABA), quien durante su infancia vivía en la Villa 31, CABA, e iba a una escuela cerca del barrio. Cuando se separaron los padres, él se mudó junto con su madre y sus hermanos a Ciudad Oculta (CO), y tuvo que empezar a trabajar en una frutería en el mercado

6 La expresión "merecer la ciudad” acuñada por el entonces Intendente de facto de la Ciudad de Buenos Aires, sintetiza una serie de prejuicios y conflictos que aluden a la representación de los pobres como una amenaza latente para aquella población que gozaba de los privilegios de vivir en el centro. Luego el término constituyó el título de la obra de Ozlak (1991) y ha sido tema de debate entre diversos investigadores que analizan la desigualdad en el acceso al espacio urbano. 
de Liniers para aportar económicamente al hogar. Además, los fines de semana, él acompañaba al padre en sus diversos trabajos eventuales y ganaba dinero como changarín, es decir ofreciendo el servicio de transportar el equipaje de los viajantes en la terminal de ómnibus "Retiro" que se encuentra en el centro de la Ciudad de Buenos Aires, lindante con el barrio Villa 31.

Abel sostenía que hubiera tenido más posibilidades laborales si hubiera contado con el título de la educación secundaria, pero creía que en su momento había perdido la oportunidad. Cuando se mudó a CO continuó yendo a trabajar de changarín a la terminal de Retiro. Durante sus 20 años tuvo diversos empleos precarios, trabajos que no eran formales y en los que cobraba por día. Entre ellos trabajó en una fábrica de bazar en la que trabajaba como repartidor, una empresa de logística en la que cargaba los camiones, una constructora para la que realizaba tareas de limpieza de obras, una fábrica de zapatos, entre otras "changas" que realizaba "para que me comieran los piojos" (Abel, comunicación personal, 2017). Abel no logró ingresar a un empleo registrado y estable como el que hubiera querido tener, pero hubo uno que mantuvo a lo largo del recorrido y fue el de changarín en la terminal. En sus palabras:

Yo soy de familia de changarines. Nunca dejé de trabajar en la terminal de changarín, hasta el día de hoy tengo familia que está ahí, viene de mi abuelo que era changarín, hasta el día de hoy trabajan, mi papá también, la otra semana fui y estaban ahí. También mis sobrinos y primos, más chicos que yo (Abel, comunicación personal, 2017).

En este sentido, si bien en su recorrido no aparece una trayectoria laboral de acumulación de experiencias que construya una identidad en base a la tarea, se observa en su discurso que la actividad, ligada a su historia familiar, adquiría un rol central en su constitución subjetiva. Según contaba, había recurrido a esa actividad informal independientemente de dónde habitara, dado que formaba parte de su historia.

Trayectorias de varones que habitan en barrios predominantemente de clase media

Otra vez, si se comparan las trayectorias del grupo anterior de varones con aquellos que terminaron sus estudios secundarios y se insertaron laboralmente, se pueden identificar especificidades de otro tipo. Los varones con mayor capital económico, social y cultural cuentan con mayores posibilidades y las decisiones deben ser comprendidas con otras lógicas. En este grupo de jóvenes, las expectativas asociadas al rol masculino están por detrás del retraso de las 
transiciones. Es decir que, si bien el rol de provisión está también presente en este sector social, sólo es asumido cuando puede ejercerse de una determinada manera. En este sentido, las dificultades de estabilización laboral pueden justificar la permanencia en el hogar de origen o el retraso de la paternidad.

Algunos jóvenes exponen entre los motivos por los cuales se quedan en la vivienda familiar la búsqueda de mayor certidumbre económica y de una realización profesional. Fernando (CABA), después de recibirse de la carrera de Economía en la Universidad de Buenos Aires, se había empleado en una empresa multinacional en la que continuaba trabajando al momento de la entrevista. Sin embargo, a sus 33 años buscaba un cambio laboral por lo que había iniciado estudios para seguir capacitándose, por lo que consideraba que su estabilización llegaría en un futuro. Fernando habitaba en la vivienda de la familia de origen, lo que consideraba como una etapa de transición hasta que decidiera realizar un movimiento residencial para vivir con su pareja. Algunos autores dieron cuenta de la tendencia al retraso de las uniones como parte de los cambios en la conformación de las familias que se registran en los últimos años en la Ciudad de Buenos Aires (Mazzeo; Ariño, 2013).

Si se focaliza la atención en sus decisiones acerca de dónde habitar, entre las perspectivas futuras llama la atención el espectro de opciones que evalúan. En la comparación con aquellos hombres que presentaban un arraigo territorial fuerte, si se analiza el relato de Fernando, se observa claramente la decisión de permanecer en la Ciudad de Buenos Aires en una comparación que incluye otras ciudades del mundo. Así lo expresaba:

Mi novia tuvo una oferta para irse a laburar a EEUU un año, la verdad que no me copa mucho, no sabía qué hacer ahí más con el tema de visas (...) Después fuimos de viaje, fuimos para Rusia y Escandinavia en las vacaciones y la verdad que sí, vos ves Finlandia, la gente, son re educados, no hay quilombo, no te matan por cruzar la calle, no te pasan por arriba en la escalera... Y te replanteás un par de cosas. Vivís más tranquilo, es otro estrés (Fernando, comunicación personal, 2013).

En su elección de un lugar para vivir, había evaluado el proyecto de irse a vivir a una ciudad del norte de Europa, pero había decidido quedarse. Si bien daba cuenta de las ventajas de vivir en ciudades del mundo con mayor calidad de vida, le preocupaba su inserción laboral. La elección del lugar para vivir en este sector social está relacionada con la búsqueda de un estilo de vida.

Pero los espacios habitados por los jóvenes van más allá de los espacios residenciales (Segura, 2012). Según relataba Tomás (La Plata), la universidad había 
sido un espacio central en su trayectoria, en el que había estudiado, trabajado y, a través de las relaciones construidas con compañeros y profesores, había ingresado tanto a la docencia universitaria como al empleo que poseía en el momento de la entrevista. Así contaba:

Yo estoy muy metido desde chico en todo el ámbito universitario, yo fui a la guardería de la Universidad de La Plata cuando mi viejo era médico de la guardería. Toda mi familia estudió en la Universidad de La Plata, mis tíos, todo el mundo. Así que es como un poco "mi casa". Y es "mi casa" también en el sentido que después empecé a dar clases en la facultad, algunas clases de Derecho Comercial como ayudante, me sumé a una cátedra, empecé a tener un poco más de "ruedo" conjuntamente con el postgrado que yo estaba haciendo y conociendo gente del ámbito de la universidad, ya a nivel docente. Me puse de novio y me casé también con una ex compañera de estudios que a la vez es abogada y es docente (...) (Tomás, comunicación personal, 2013).

Si se compara con el relato de Abel, del grupo de jóvenes de barrios relegados, para quien uno de los espacios ordenadores de su trayectoria había sido la terminal de micros, en el caso de Tomás, también aparece un espacio que permanece constante a lo largo del recorrido y articula su trayectoria, la Universidad Pública. Es un espacio articulador de su recorrido tanto laboral como familiar. A diferencia de Abel, en este caso el espacio se había constituido en una fuente multiplicadora de recursos.

A lo largo del capítulo aparecen puntualizadas algunas de las relaciones que establecen los jóvenes con los lugares que habitan, principalmente los residenciales, pero también otros de los espacios que organizan sus trayectorias sociales. A partir de las diferencias entre mujeres y varones de la muestra, se evidencian algunas de las características de las transiciones a la vida adulta de aquellos que habitan en distintos tipos de barrio para visualizar las diferentes gramáticas de la juventud. Este concepto consiste en una estilización de los tipos ideales de trayectorias que sirve de base para establecer una mirada global sobre la juventud actual.

\section{Debate}

Los resultados de la investigación indican que las mujeres que habitan en barrios predominantemente de clase media tienen un amplio espacio para la socialización y la movilidad independiente donde los límites van más allá de 
la región Sur. Como parte de este proceso, entre las mujeres integradas en los flujos mundiales, la extensión del estatus de la juventud, la movilidad independiente y el aplazamiento de la maternidad están disponibles y son opciones socialmente valoradas.

Sin embargo, para las mujeres de barrios relegados, el barrio es el espacio más importante para la socialización y la construcción de identidades. Para aquellas mujeres que viven en barrios menos privilegiados, los dilemas se configuran en redes ubicadas territorialmente, donde las subjetividades se organizan según los formatos de reproducción familiar. En estos casos, la maternidad y las tareas de cuidado se convierten en las principales actividades y las expectativas que delinean las transiciones juveniles.

Como contracara del rol de cuidado entre las mujeres, el rol de proveedor del varón prevalece como modelo normativo y se ven sus efectos cuando éste no puede cumplirse. Entre los varones de barrios relegados las transiciones, tanto familiares y laborales como residenciales, también comenzaban de forma precoz, durante la adolescencia. En los relatos de los entrevistados de menores recursos, se relacionó la transición familiar temprana con la necesidad de constituirse en proveedores de ingresos, entre los cuales el trabajo se constituye en una de las formas posibles de lograrlo (Arancibia, 2018).

Para los jóvenes varones de barrios predominantemente de clase media, el modelo normativo de la provisión se mantiene constante, repercutiendo en las decisiones laborales y de conformación de hogares propios. Estos jóvenes, al igual que sus pares mujeres, aplazan el inicio de las transiciones a la vida adulta, y desarrollan biografías de "elección", vinculadas a arraigos simbólicos y estatutarios siguiendo el patrón hegemónico.

\section{Conclusiones}

En América Latina, la desigualdad se manifiesta en la coexistencia de áreas de gran desarrollo económico con otras de grandes privaciones, configurando espacios urbanos altamente fragmentados. La informalidad de los barrios se expresa a través de distintas denominaciones tales como: villas, favelas, cantegriles, entre otros. La vigencia y profundización de la fragmentación ha confluido en procesos de segregación urbana dando lugar a la polarización de las trayectorias de las y los jóvenes. La desigualdad espacial afecta de forma diferente a las mujeres y a los varones. Entre las primeras, especialmente en relación con sus perspectivas de participación económica, como resultado de la distribución inequitativa de las responsabilidades en torno al cuidado de 
las familias. Entre los varones, las dificultades de acceder al mercado laboral y el tipo de ocupaciones a las que acceden por las redes de sociabilidad barriales, en algunos casos pueden estar relacionadas con el consumo problemático de sustancias y actividades delictivas. En un contexto de gran inestabilidad y vulnerabilidad, el fuerte vínculo de pertenencia al barrio marcó trayectorias situadas localmente, constituyendo una alternativa a las gramáticas hegemónicas de la juventud, con escaso reconocimiento social y fuerte estigmatización. El campo de los estudios de juventud está en deuda con la perspectiva de género (Miranda; Arancibia, 2017), ya sea enfocando en feminidades, masculinidades y otredades.

Con el objetivo de aportar al debate teórico sobre la construcción de la desigualdad social entre la juventud, en el texto se trabajó en el análisis de trayectorias sociales de hombres y mujeres a partir de los resultados del Programa Gramáticas de la Juventud. A través de entrevistas retrospectivas realizadas con personas jóvenes que alcanzaron la mayoría de edad durante una de las peores crisis sociales del país, pero que luego fueron contemporáneas al crecimiento económico y la protección social de principios de siglo veintiuno, se presentó el debate sobre la intersección entre juventud, espacio y géneros, en tanto claves para la reflexión sobre la construcción social de las gramáticas juveniles.

El punto de partida del análisis abordó una serie de preguntas sobre el espacio, el arraigo y la construcción de identidades femeninas y masculinas. La contribución original se basó en relacionar el arraigo al lugar con las trayectorias juveniles y la fragmentación territorial. A su vez, se buscó echar luz acera de la matriz heterosexual, la estructura patriarcal que define ciertos patrones de género. Evidenciando un fuerte contraste, los hallazgos del trabajo de campo mostraron cómo en el esquema patriarcal de relaciones de género se desarrollan diferentes formas de arraigo, construidas en diferentes escalas espaciales, con una fuerte influencia en los recorridos biográficos de las y los jóvenes.

En efecto, los asuntos relativos a la educación y el trabajo se encuentran enraizados en decisiones vitales sobre la forma de organización familiar, la unión conyugal, la maternidad/paternidad, en las que se expresa la ideología de género vigente en una sociedad. Sin embargo, como sostiene Segato "es necesario escudriñar a través de las representaciones, las ideologías, los discursos acuñados por las culturas y las prácticas de género para acceder a la economía simbólica que instala el régimen jerárquico y lo reproduce" (Segato, 2010: 14).

Con el foco puesto en las diferentes escalas espaciales en las que suceden las trayectorias juveniles de mujeres y varones, se pudo ver la coexistencia de diferentes formas de arraigo y sus efectos en la construcción de gramáticas 
juveniles diferenciadas. Estas gramáticas, adquieren también status diferenciados en los distintos espacios habitados por las y los jóvenes. De esta forma, en los testimonios pudo observarse la contradicción entre los valores hegemónicos asociados a las actividades juveniles, y los valores subalternos construidos de forma situada en una dinámica social disidente, comunitaria.

La programación pública de políticas de juventud y empleo pocas veces toma en consideración las particularidades locales de la población joven. Los programas enfocan a un/a individuo/a aislado, de forma comparativa con la gramática oficial, en donde los y las jóvenes de clases medias y altas se desempeñan "reflexivamente". Los resultados de investigación brindan elementos válidos para construir alternativas de programación que incluyan a los factores comunitarios, trabajen en contrarrestar la dinámica de la segmentación, tomen en cuenta los factores asociados a la estigmatización, en dirección a la promoción de la justicia social, el reconocimiento de la identidad y la afirmación de la participación entre las y los jóvenes.

\section{Referencias}

AGUIRRE, Rosario; BATTHYÁNY, Karina; GENTA, Natalia; PERROTTA, Valentina.

"Los cuidados en la agenda de investigación y en las políticas públicas en Uruguay". Iconos. Revista de Ciencias Sociales, n. 50, 2014, pp. 43-60.

ARANCIBIA, Milena Maia. (2018) Desigualdad espacial, género y acceso a la vivienda: un estudio sobre trayectorias juveniles en el AMBA, 1999-2017. Tesis de Doctorado, Universidad de Buenos Aires. CABA.

MAZZEO, Victoria; ARIÑO, Mabel. Estrategias familiares de las generaciones post-70 en la Ciudad de Buenos Aires: jóvenes viejos o niños eternos? Población de Buenos Aires, año 10, núm. 17, 2013, pp. 65-76.

BAYÓN, María Cristina. La integración excluyente. Experiencias, discursos y representaciones de la pobreza urbana en México. Mexico, Bonilla Artigas 2015.

BAYÓN, María Cristina; SARAVÍ, Gonzalo A. "Place, class interaction, and urban segregation: experiencing inequality in Mexico City". Space and Culture, v. 21, n. 3, 2017, pp. 291-305.

BENDIT, René. Los jóvenes y el futuro: procesos de inclusión social y patrones de vulnerabilidad en el mundo global. BENDIT, René; HAHN, Marina; MIRANDA, Ana. (Eds.). Transiciones juveniles: procesos de inclusión social y patrones de vulnerabilidad en un mundo globalizado. Buenos Aires, Prometeo, 2008.

BENDIT, René; MIRANDA, Ana. "La gramática de la juventud: un nuevo concepto en construcción”. Última Década, v. 25, n. 46, 2017, pp. 4-43. 
BENDIT René; MIRANDA, Ana. Turning thirty: youth transition process in Argentina in 21 century. Journal of Applied Youth Studies, v. 1, n. 3, 2016, pp. 96-108. http://cayr. info/jays-v-1-n-3/

BOURDIEU, Pierre. El sentido práctico. Argentina, Siglo XXI de España Editores, 2008. CERBINO, Mauro. El lugar de la violencia: perspectivas críticas sobre pandillerismo juvenil. Quito, Taurus, 2012.

CHAVES, Mariana. (2005). Juventud y espacios urbanos en la ciudad de La Plata. Doctoral dissertation, Tesis de doctorado, Facultad de Ciencias Naturales y Museo, UNLP, La Plata. Mimeo.

CONNELL, Raewyn. Southern theory: The global dynamics of knowledge in social science. Allen \& Unwin, 2007.

CRAVINO, María Cristina. Vivir en la villa: relatos, trayectorias y estrategias habitacionales. Los Polvorines: Universidad Nacional General Sarmiento, 2009.

CUERVO, Hernán; WYN, Johanna. "Reflections on the use of spatial and relational metaphors in youth studies". Journal of Youth Studies, v. 17, n. 7, 2014, pp. 901-915.

CUERVO, Hernán; WYN Johanna. "A longitudinal analysis of belonging: temporal, performative and relational practices by young people in rural Australia”. Young, v. 25, n. 3, 2017, pp. 1-16. doi:10.1177/1103308816669463.

FARRUGIA, D. Space and place in studies of childhood and youth. In: WYN, Johanna; CAHILL, Helen. (eds.): Handbook of Children and Youth Studies. Springer, Singapore, 2014.

FRAIMAN, R. Y ROSSAL, M. (2009). Si tocás pito te dan cumbia. Esbozo antropológico de la violencia en Montevideo. Montevideo: Ministerio del Interior/PNUD/AECID.

FRASER, Nancy. Contradictions of capital and care. New Left Review, n. 100, 2016, pp. 99-117.

GRIMBERG, Silvia. et al. Carcova is love: Becoming youth in the slums of the global south. En CUERVO, Hernán; MIRANDA, Ana. Eds. Youth, Inequality \& Social Change in the Global South. Springer for the series Perspectives on Children and Young People, Springer, 2019.

HOPKINS, Peter. Scales of young people's lives. In: WYN, Johanna; CAHILL, Helen. (eds.) Handbook of Children and Youth Studies, Springer, 2015.

KESSLER, Gabriel. Movilidades laterales: delito, cuestión social y experiencia urbana en la periferia de Buenos Aires. Revista de Ciencias Sociales, v. 25, n. 31, 2012, pp. 37-58.

MACDONALD, Robert; SHILDRICK, Tracy; WEBSTER, Colin; SIMPSON, Donald. Growing up in poor neighbourhoods: the significance of class and place in the extended transitions of 'socially excluded' young adults. Sociology, v. 39, n. 5, 2005, pp. 873-891. 
MARCO NAVARRO Flavia. El Vivir Bien de la niñez y la adolescencia y el ejercicio de derechos. En FARAH, Ivonne; TEJERINA, Veronica. (coord.). Vivir bien: infancia, género y economía: entre la teoría y la práctica. La Paz. CIDES-UMSA/UNICEF, 2013. MIRANDA, Ana; ARANCIBIA, Milena. El futuro está incompleto: La construcción de trayectorias laborales sobre principios de siglo 21. Revista Trabajo y Sociedad, n. 28, 2017, pp. 195-217.

OSZLAK, Oscar. Merecer la ciudad: los pobres y el derecho al espacio urbano. Buenos Aires, Cedes, 1991.

PERELMAN, Laura; VARGAS, Patricia. Imputaciones morales y precarización laboral entre los jóvenes trabajadores de una fábrica siderúrgica en la Argentina. Trabajo y sociedad, n. 21, 2013, pp. 403-413.

REGUILLO CRUZ, Rossana. Emergencia de culturas juveniles: estrategias del desencanto. Bogotá, Norma, 2000.

RODRÍGUEZ, María Carla. et al. (2007) Producción social del hábitat y políticas en el Área Metropolitana de Buenos Aires: historia con desencuentros, Documentos de Trabajo No 49, Instituto de Investigaciones Gino Germani, Universidad de Buenos Aires.

RODRÍGUEZ ENRÍQUEZ, Corina; MARZONETTO, Gabriela. Organización social del cuidado y desigualdad: el déficit de políticas públicas de cuidado en Argentina. Perspectivas de Políticas Públicas, v. 4, n. 8, 2015, pp. 105-134.

SALAS Minor Mora; PEREZ SÁINZ, Juan Pablo. (2018) El desafío de la inclusión laboral de jóvenes en barrios urbano-marginales en Centroamérica: más allá de las políticas de capacitación para el empleo. En Córica, A, Freytes, A y Miranda, A. (comp.) Entre la educación y el trabajo: la construcción cotidiana de las desigualdades juveniles en América Latina. Buenos Aires, CLACSO.

SARAVÍ, Gonzalo. A. Segregación urbana y espacio público: los jóvenes en. Revista de La CEPAL, 83, 2004, pp. 33-48.

SARAVÍ, Gonzalo. A. Juventudes fragmentadas. Socialización, clase y cultura en la construcción de la desigualdad. México, FLACSO México/CIESAS, 2015.

SAVAGE, Mike. The politics of elective belonging. Housing, Theory and Society, v. 27, n. 2, 2010, pp. 115-135.

SAVAGE, Mike; BAGNALL, Gaynor; LONGHURST, Brian. Globalization and belonging. London, Sage, 2005.

SEGATO Rita Laura. Las estructuras elementales de la violencia ensayos sobre género entre la antropología, psicoanálisis y derechos humanos. Buenos Aires, Prometeo, 2010.

SEGURA, Ramiro. Elementos para una crítica de la noción de segregación residencial socio-económica: desigualdades, desplazamientos e interacciones en la periferia de La Plata. Quid 16: Revista del Área de Estudios Urbanos, n. 2, 2012, pp. 106-132. 
VALENZUELA ARCE, J. M. El sistema es antinosotros: culturas, movimientos y resistencias juveniles. Mexico, Gedisa, 2015.

WARD, M. R. Michael. "I'm a Geek I am": Academic achievement and the performance of a studious working-class masculinity. Gender and Education, v. 26, n. 7, 2014, pp. 709-725.

WARR, Deborah. The ambivalent implications of strong belonging for young people living in poor neighborhoods. WYN, Johanna; CAHILL, Helen. (eds.): Handbook of Children and Youth Studies. Singapore, Springer, 2015.

WORTH, Nancy. Youth, relationality, and space: conceptual resources for youth studies from critical human geography. WYN, Johanna; CAHILL, Helen. (Eds.): Handbook of Children and Youth Studies. Singapore, Springer, 2015.

Recebido em: 27/05/2019

Aprovado em: 06/12/2019

\section{Como citar este artigo:}

ARANCIBIA, Milena e MIRANDA, Ana. La construcción social de gramáticas juveniles: reflexiones sobre la desigualdad a través de estudios longitudinales. Contemporânea - Revista de Sociologia da UFSCar, v. 9, n. 3, set.- dez. 2019, pp. 823-846. 